\section{Gastrointestinal mucormycosis resulting in a catastrophic out- come in an immunocompetent patient}

\author{
Suresh J. Antony, ${ }^{1}$ Monisha S. Parikh, ${ }^{1}$ \\ Ruben Ramirez, ${ }^{2}$ Bruce Applebaum, ${ }^{2}$ Glen \\ Friedman, 2 Jennifer Do ${ }^{1}$ \\ 'Department of Medicine, Division of \\ Infectious Diseases, Texas Tech \\ University Health Sciences Center, EI \\ Paso; ' Department of Pathology, \\ Gastroenterology and Surgery, Las \\ Palmas Medical Center, EI Paso, TX, USA
}

\begin{abstract}
We present a case of a middle-aged female who was admitted to the hospital with a respiratory infection and subsequently developed an acute surgical abdomen secondary to a perforated viscous. She was found to have mucormycosis of the intestinal tract and eventually succumbed to the sequelae of the infection.
\end{abstract}

\section{Introduction}

Mucormycosis is a rare fungal infection that typically involves the rhino-cerebral area with a high morbidity and mortality. Involvement of the gastrointestinal tract with perforation is rare and may be related to dissemination of the disease. The current antifungal treatment has been shown to be inadequate with variable outcomes and is often instituted late in the disease with dismal outcomes.

\section{Case Report}

This is a 58-year-old Hispanic female with a past medical history of trigeminal neuralgia and hyperlipidemia, who developed an upper respiratory tract infection, which lasted for approximately one week prior to admission. She complained of a cough, occasional shortness of breath, and low-grade fevers. She was seen by her private physician prior to admission and was given decadron $40 \mathrm{mg}$ i.m and a prescription for antibiotics. She did not fill the prescription for the antibiotics immediately. She then noted that her shortness of breath and cough were getting worse. She was seen in the emergency where she was diagnosed with an acute airway obstruction secondary to acute epiglottitis. She was emergently intubated and transferred to the intensive care unit where she was noted to be hypotensive (BP $90 / 76 \mathrm{mmHg}$ ) and hypoxemic. In addition to the ventilator support, her intensive care unit (ICU) therapy required vasopressors.

The chest $\mathrm{x}$-ray showed extensive bilateral peri-hilar consolidation and infiltrates. The computed tomography (CT) scan of the chest revealed diffuse bilateral interstitial and alveolar infiltrates with bilateral lower lobe air space consolidation. A CT scan of the neck revealed diffuse swelling of the soft tissues with loss of the fat planes throughout the pharyngeal region with marked thickening of the retropharyngeal soft tissue. There was no evidence of loculated fluid collections to suggest an abscess. She had minimal cervical adenopathy that was noted.

Her laboratory results revealed leukocytosis $\left(15.8 \times 10^{9} / \mathrm{L}\right)$, a borderline hematocrit level (44\%), and a normal platelet count $\left(170.5 \times 10^{9} / \mathrm{L}\right)$. Her metabolic panel was unremarkable with a normal sodium (139 mEq/L), and normal renal function (blood urea nitrogen $14 \mathrm{mg} / \mathrm{dL}$ and creatinine $1.1 \mathrm{mg} / \mathrm{dL}$ ). Her liver function tests revealed a normal alanine aminotransferase (ALT 31 IU/L) and aspartate aminotransferase (AST 17 IU/L). Her arterial blood gas showed significant acidosis with a $\mathrm{pH}$ of 7.03, a PaCO2 level of $46 \mathrm{mmHg}$, and a $\mathrm{PaO} 2$ of $46 \mathrm{mmHg}$. Her blood cultures were negative.

\section{Hospital course}

The patient remained intubated in the ICU for approximately one week secondary to her acute respiratory failure. She underwent an endoscopic evaluation on her admission to the hospital that was unremarkable for any unusual pathology. Following an extensive workup for infection, which was unremarkable, she was started on intravenous solumedrol ( $40 \mathrm{mg}$ q8h) for approximately 14 days. Chest x-rays performed on multiple occasions continued to show bilateral infiltrates. Multiple workups for a source of infection were all negative including blood, urine, and sputum cultures. Serology for influenza, parainfluenza, Brucella, tuberculosis, Bordetella pertussis, and atypical pathogens such as the Mycoplasma, Legionella, and Chlamydia pneumoniae were all negative. During the hospitalization she was placed on broad-spectrum antibiotics including antifungal (fluconazole $400 \mathrm{mg}$ daily) as well.

The patient was eventually extubated and transferred to the medical floor of hospital day 10. However, while on the floor she developed recurrent respiratory distress necessitating reintubation and ICU readmission. She underwent a bronchoscopy that did not reveal any abnormalities. The bronchoalveolar lavage and lung biopsy were normal prior to the dissemi-
Correspondence: Suresh J. Antony, Department of Medicine, Division of Infectious Diseases, Texas Tech University Health Sciences Center, 1205 N. Oregon, El Paso 79902, TX, USA.

Tel.: +1.915.533.4900 - Fax: +1.915.533.4902

E-mail: suresh.antony@att.net

Key words: Mucormycosis; gastrointestinal tract; gastrointestinal bleeding.

Contributions: the authors contributed equally.

Conflict of interest: the authors declare no potential conflict of interest

Received for publication: 22 May 2015.

Revision received: 18 July 2015 .

Accepted for publication: 18 July 2015 .

This work is licensed under a Creative Commons Attribution NonCommercial 3.0 License (CC BYNC 3.0).

(O)Copyright S. J. Antony et al., 2015

Licensee PAGEPress, Italy

Infectious Disease Reports 2015; 7:6031

doi:10.4081/idr.2015.6031

nation of the infection. She then developed evidence of a lower GI hemorrhage. An upper endoscopy to investigate the source of hemorrhage was non-contributory and a biopsy was not done. A planned colonoscopy could not be performed when the patient developed hypotension. A subsequent KUB showed massive pneumoperitoneum, requiring an emergent laparotomy.

Two segments of small bowel $(40 \mathrm{~cm}$ in aggregate length), a right hemicolectomy specimen $(60 \mathrm{~cm}$ segment of small bowel with $20 \mathrm{~cm}$ segment of large bowel), and omental tissue were evaluated by Pathology. The serosal surface of the bowel showed a variable green discoloration with areas of adherent fibrinopurulent exudate associated with multiple small bowel perforations and a $4 \mathrm{~cm}$ long cecal perforation. The mucosa showed several shallow ulcers and areas of edema alternating with marked thinning. Microscopic sections showed innumerable invasive fungal elements throughout the submucosal and peritoneal tissue with many areas of vascular invasion. The fungal hyphae were broad, undulating, ribbonlike, and showed branching at random angles and rare septae. Vascular thrombosis, hemorrhage, acute inflammation, and necrosis were also seen. The fungal morphology was most consistent with Mucormycosis, although Aspergillus was considered in the differential diagnosis (Figure 1). Specimens from two subsequent surgeries, comprising multiple segments of small bowel that totaled $50 \mathrm{~cm}$ in aggregate length and a $5 \mathrm{~cm}$ long segment of large bowel, all showed similar gross and 
microscopic findings.

She was started on liposomal amphotericin $B$ and micafungin after discontinuation of the fluconazole. Additional intestinal resections were performed with each subsequent laparotomy. At the final laparotomy 6 days after the initial operative intervention, the patient had near complete intestinal infarction. With evidence of progressive multiorgan failure, ongoing shock, and diffuse intestinal ischemia and infarction; the patient's condition was deemed terminal. Discussions with her family led to DNR status and withdrawal of care. The patient succumbed to the illness.

\section{Discussion and Conclusions}

Mucormycosis can affect any organ system. With gastrointestinal mucormycosis infections, the most common site is the stomach, followed by colon and ileum. ${ }^{1}$ The disease process can mimic ischemic colitis and is commonly first diagnosed (as in this case) by pathology, not by microbiology.

Mucormycosis is a rare and often life-threatening disease that is commonly seen in patients who have immunodeficiency syndromes such as malignancies, solid organs transplants, long term steroid therapy, and poorly controlled diabetes. There have been some recent changes in the taxonomy of some of these invasive fungal pathogens. Fungal pathogens that are associated with mucormycosis and now classified under the phylum Glomeromycota. ${ }^{2-4}$ Mucormycosis is most often located in the head and neck area, lungs, skin, and occasionally can be found disseminated in the gastrointestinal tract. ${ }^{3}$ The prognosis for most patients with mucormycosis is extremely poor with a high morbidity and mortality.,5

Mucormycosis has been reported in the pediatric literature causing necrotizing enterocolitis. ${ }^{4}$ In transplant recipients, the gastrointestinal tract is involved, especially in liver transplant patients. They typically present with non-ischemic colitis that resolves spontaneously within 24 to 48 hours. Occasionally the disease does progress resulting in significant morbidity and mortality. ${ }^{3,5}$

Several clinical forms of mucormycosis are recognized. The tendency of this fungus to invade the blood vessel walls often produces a disseminated infection. A hemo-phagocytic syndrome usually complicates the disseminated disease. ${ }^{3,5}$

Most cases of mucormycosis occur as a result of environmental exposure. In a study done by Rammaert et al., a total of 169 cases of healthcare-associated mucormycosis were studied between 1970 and 2008. The major underlying disease processes in these cases were solid organ transplant in $24 \%$, diabetes mellitus in $22 \%$, and prematurity in $21 \%$. The most commonly affected areas were the skin in $57 \%$ and the gastrointestinal tract in $15 \%$. The portal of entry was thought to be surgery and the presence of medical devices, such as catheters were implicated. Only seventy five percent of the patients were cultured, of which $92 \%$ were positive for the fungus. The histopathological examinations in these tissues were positive in $95 \%$ of the patients. The most frequent species seen in $43 \%$ of the patients was Rhizopus. ${ }^{6}$

In a study that was done by Mignogna et al., an extensive database analysis of immunocompetent, healthy patients with mucormycosis infections was done between 1978-2009 to better understand the common locations and presentations. This resulted in 212 patients studied. India had the largest percentage of patients, 94 patients (44.3\%), with the most
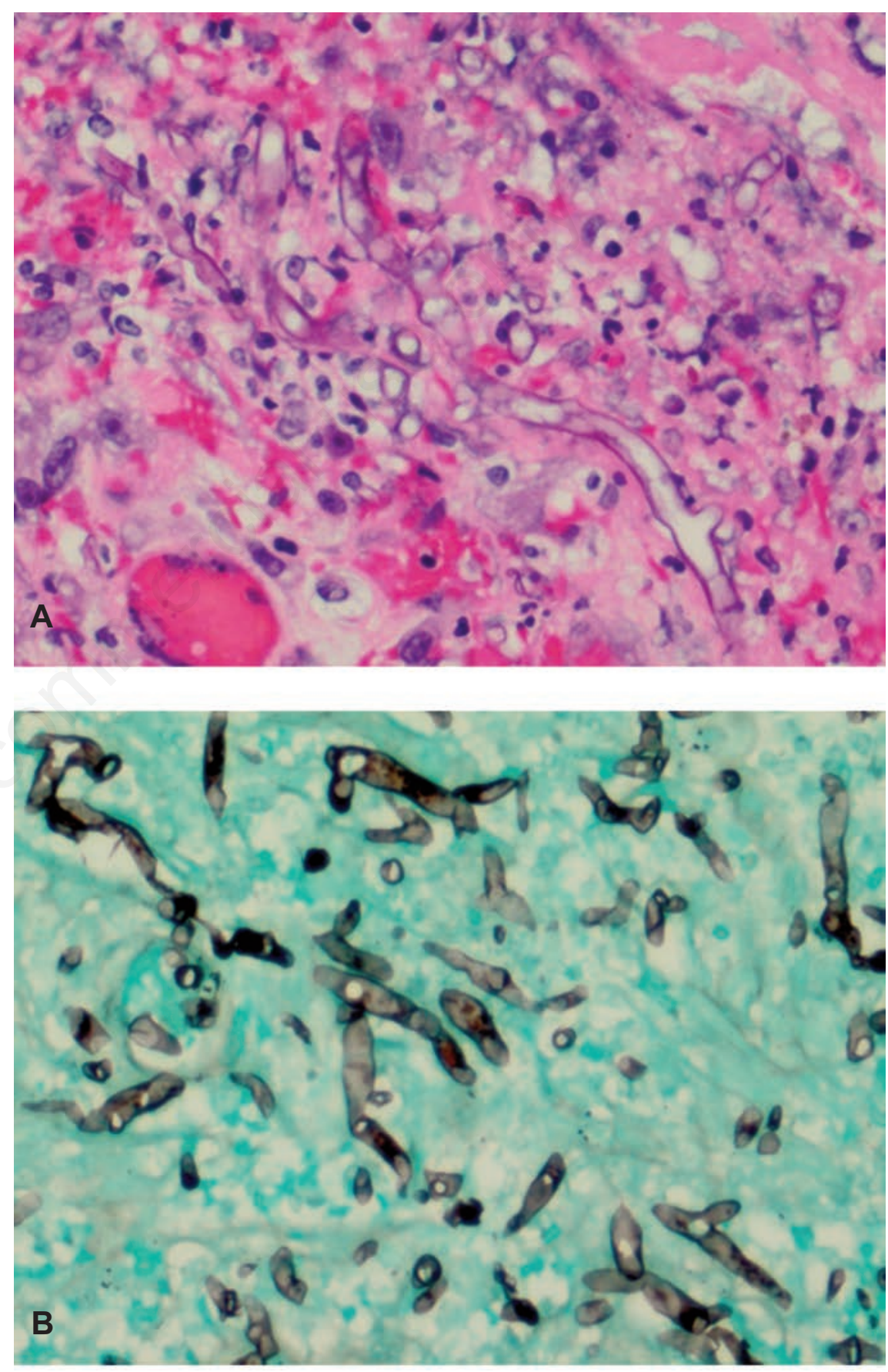

Figure 1. A) Mucormycosis in ischemic bowel (Hematoxylin \& Eosin stain); B) mucormycosis (Gomori methenamine-silver stain). 

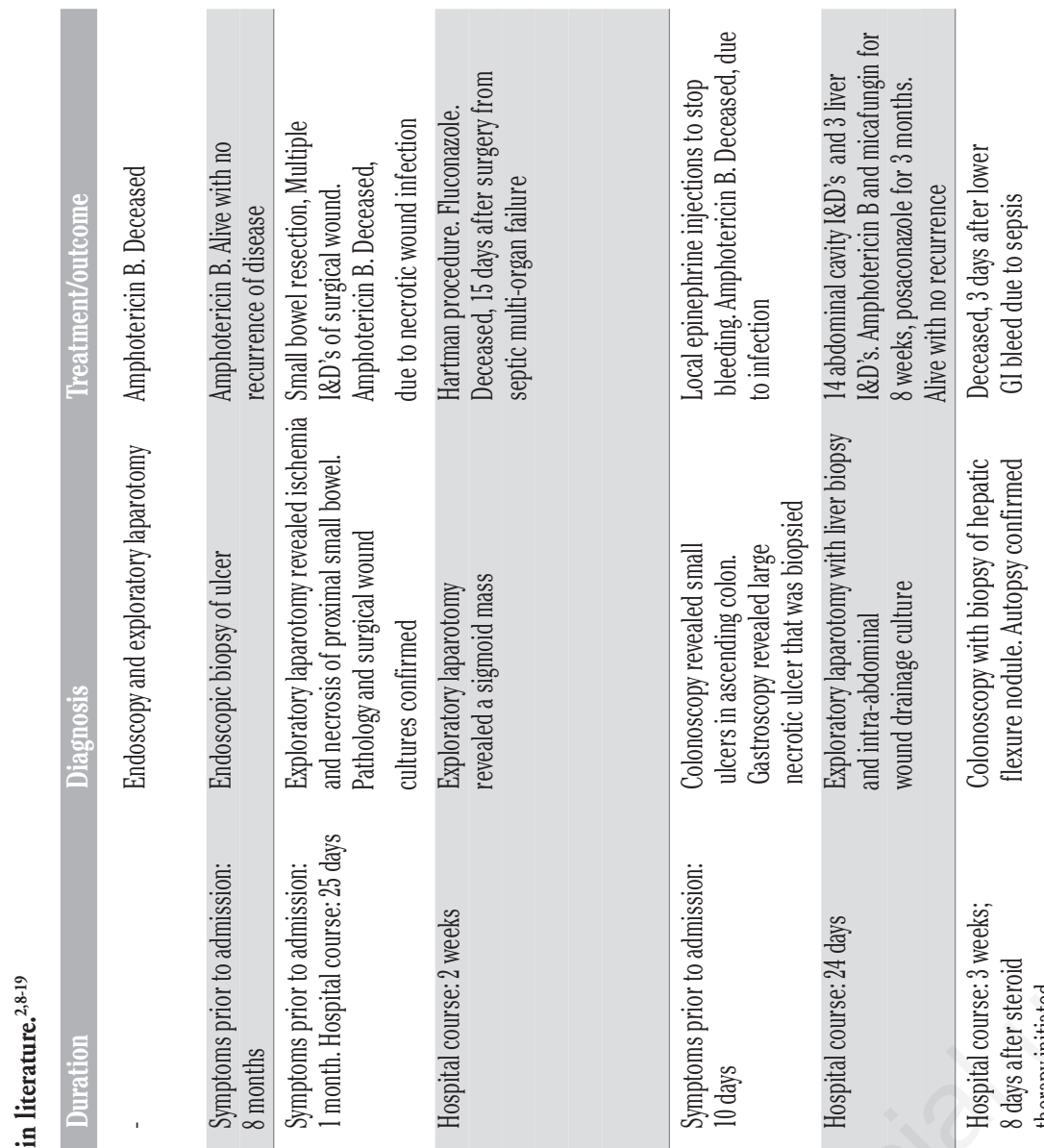

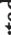

踏

部

롤

政

(1)
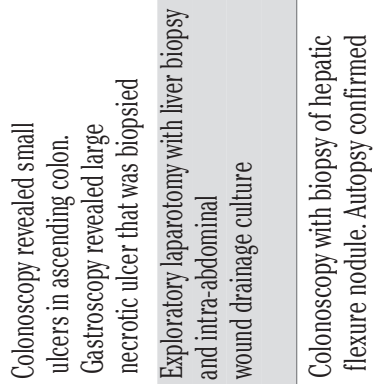

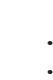

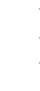

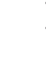

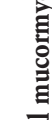

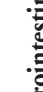

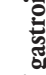

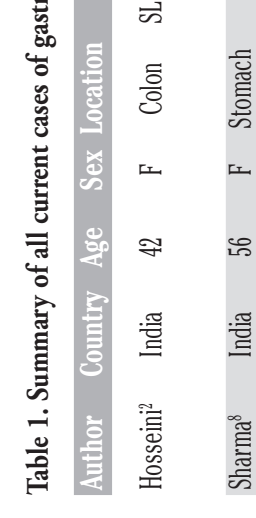$$
1
$$$$
\text { 言的地 }
$$

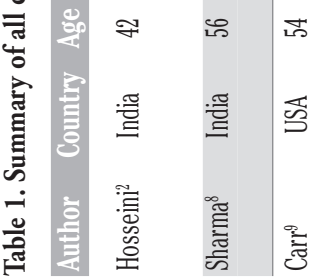
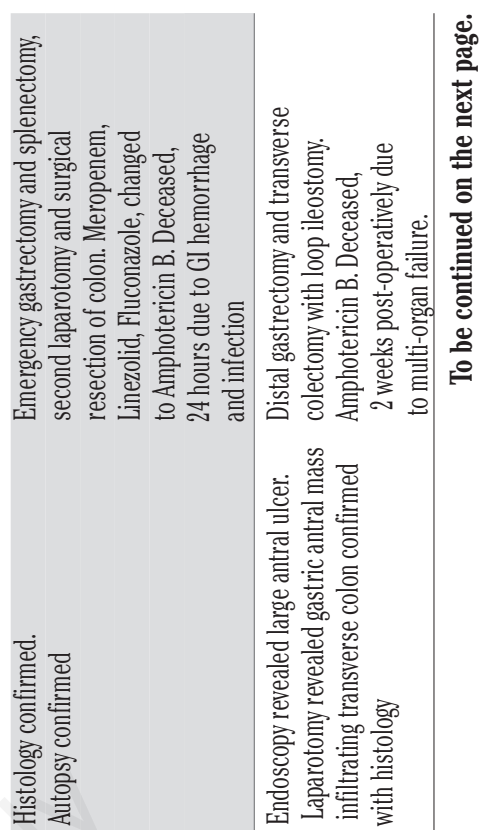

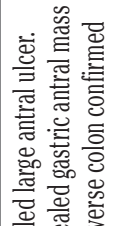

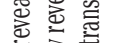

巳

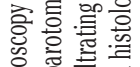

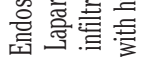
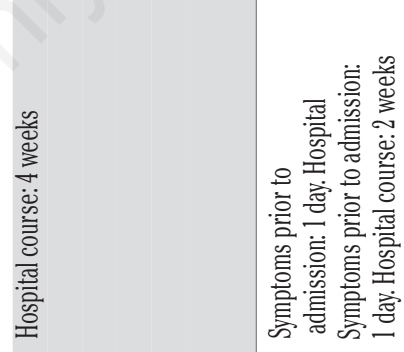

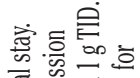

क्ष

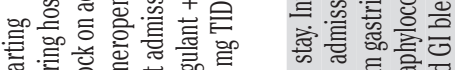

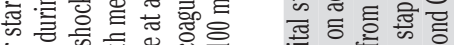

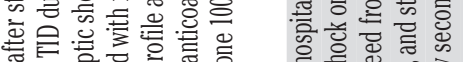

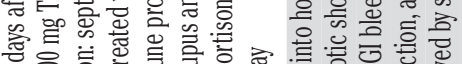

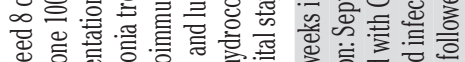

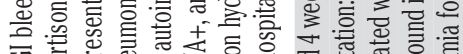

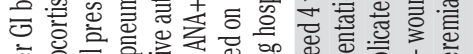

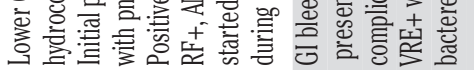

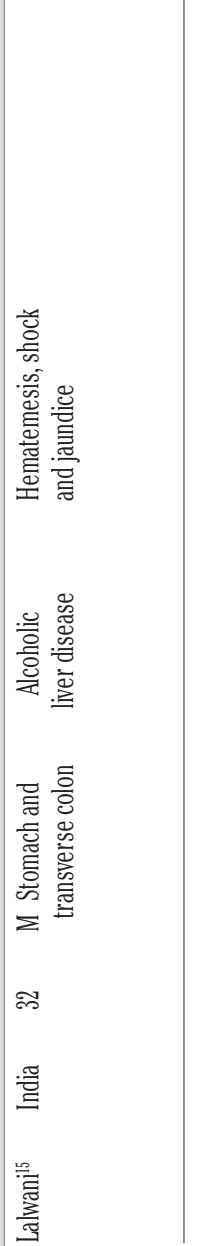

$\stackrel{0}{\frac{0}{2}}$

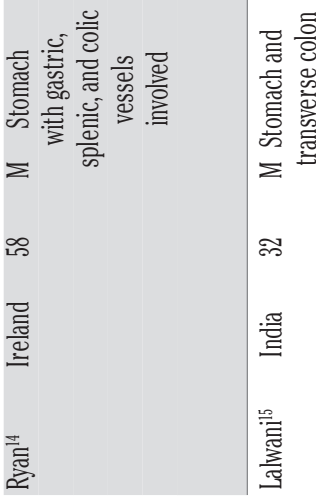




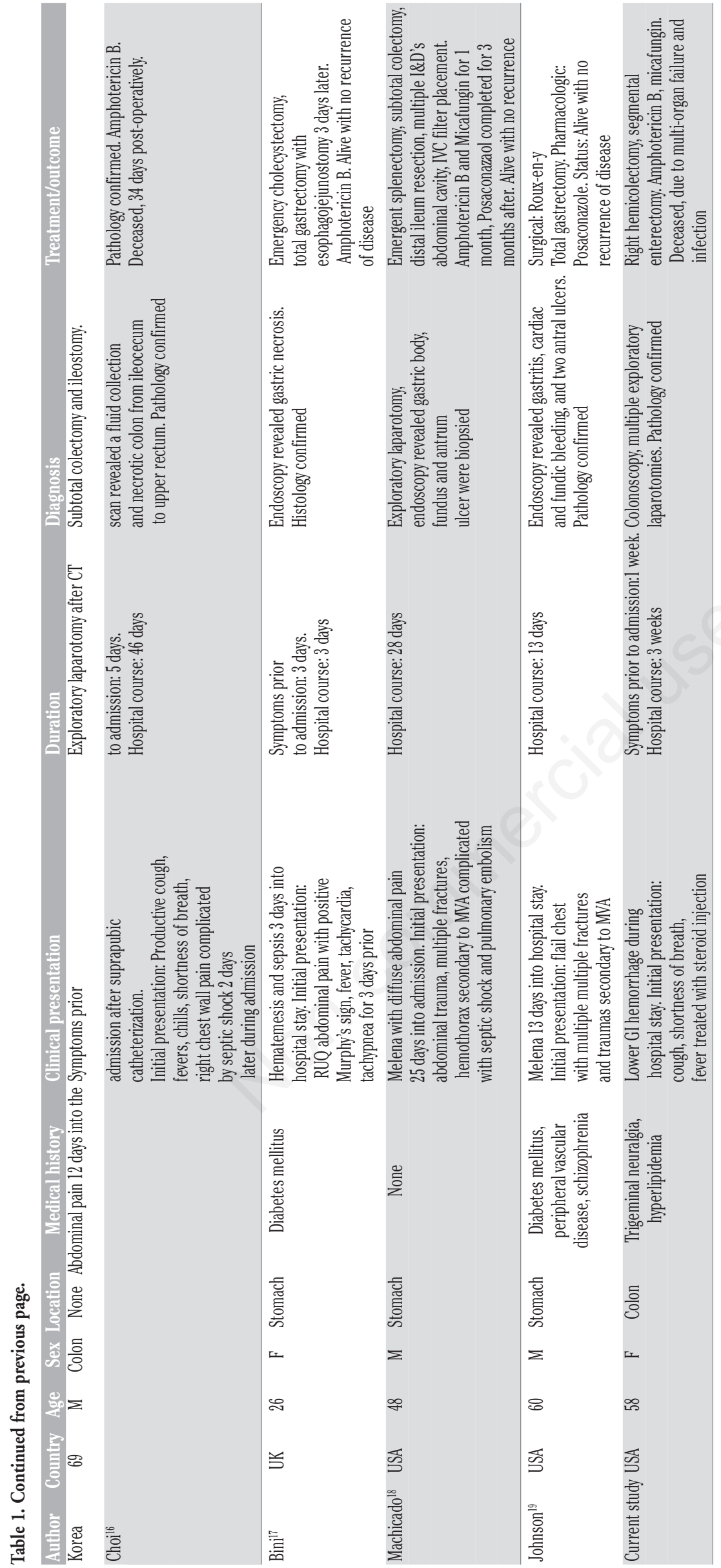

common clinical presenting form reported as a cutaneous or subcutaneous abscess in $42 \%$ of the patients. There were only five patients who were immunocompetent where the fungus was diagnosed at the sinus and responded well to liposomal amphotericin B. ${ }^{7}$

We summarized all the current cases reported in the literature of mucormycosis affecting the gastrointestinal tract in immunocompetent adults. Only fourteen cases have been reported that meets this criteria and are summarized in Table 1.,8-19 The earliest reported case was in 1998. Prior to our case, only four cases have been reported in the United States. It is important to note the similarities between these cases, including their clinical presentation.

In almost every case, patients were admitted to the hospital for a different underlying cause, such as concerning respiratory symptoms, septic shock, or trauma. The patients then presented with a GI bleed, either upper or lower, depending on the site of infection (stomach versus colon). This seems to be a presenting sign of mucormycosis in these patients and should be considered as an early differential diagnosis in all future patients with similar presentations due to the aggressiveness of the infection. Following the GI bleed, these patients deteriorated quickly due to septic shock and multiorgan failure with only five of the fourteen patients in the reported cases surviving. GI mucormycosis is more common in immunosuppressive patients, but it should be noted that in three of the cases the patients had recent steroid exposure. These patients were given steroids either prior to their hospital admission or during their hospital stays that could be thought to be an inciting factor for the infection. In all three cases of steroid use, all three patients succumbed to the infection.

It is also possible that the GI hemorrhages as a presenting symptom are related to stress ulcers from the initial sepsis, trauma, or symptoms that each patient presented with. The wide use of proton pump inhibitors and anticoagulant use in patients with extended hospital stays should also be postulated as a compounding factor related to the massive GI bleeds that presented in each patient.

We postulate that our case has a similar pathophysiology as identified in Table 1 with the primary site of infection being the parapharyngeal region, a common location of murcormycosis, that either began endogenously or as a result of her recent steroid use. This is why we suspect our patient presented with respiratory symptoms and subsequent respiratory distress before the infection invaded blood vessels walls and disseminated to the GI tract causing ulceration and perforation. A bronchoscopy with a bronchial alveolar lavage and biopsy were negative prior to the spread of infection that did not definitely yield a source 
of infection. All cultures were negative and a diagnosis was made by a colon tissue culture post-mortem. Biopsies were not obtained during the upper endoscopy when investigating the source of the GI bleed. For these reasons, we can only postulate the source and route of infection in this case. This suspected route of infection would be in line with the known pathophysiology of mucormycosis and with all of the previous cases that have been reported in literature.

Mucormycosis infections are difficult to diagnosis and treat due to the variable presentations, delayed diagnosis, and aggressiveness of the disease course. Skiada et al. summarized the first set of guidelines addressing the management of mucormycosis infections in 2012. There are no current IDSA grade A or I classifications to guide antifungal therapy for the successful treatment of mucormycosis. The current management is largely based on case reports, animal studies, and in vitro data. $^{20}$

Current ECIL 3 recommendations that were developed using the IDSA recommendation model and the following recommendations are for the first-line treatment in mucormycosis infections. A combination of antifungal therapy, surgery, and control of the underlying conditions should be employed for the primary management of mucormycosis, as a grade AII recommendation. The use of liposomal amphotericin $B$ at $5-10 \mathrm{mg} / \mathrm{kg}$ and the use of amphotericin B lipid complex (ABLC) at 5-7.5 mg/kg are recommended as grade BII and BIII, respectively. Amphotericin $B$ deoxycholate and amphotericin B colloidal dispersion are both grade CII recommendations. The use of Posaconazole $400 \mathrm{mg}$ BID is a grade CIII recommendation. The use of combination therapy is also a grade CIII recommendation. ${ }^{20}$

The use of surgery in combination with antifungal therapy and underlying disease management has been recommended, but is largely based on the location and depth of the infection. For rhino, orbital, cerebral, and soft tissue infections, surgery is recommended with a grade AII recommendation in these cases. Surgical intervention for localized pulmonary lesions is a grade BII recommendation. Surgical intervention for disseminated infections is a grade CIII recommendation. ${ }^{20}$ These recommendations do not specifically address GI infections or invasive infections, as in our case.

For the secondary treatment of mucormycosis due to treatment failure, intolerance, and maintenance, the following recommendations have been given. Posaconazole $400 \mathrm{mg}$ BID is a grade BII recommendation for cases refractory to treatment, and maintenance therapy with Posaconazole is a grade BIII recommendation. Combination therapy with lipid amphotericin $B$ and caspofungin may be a useful salvage treatment with a grade BII recommendation. Combination therapy with lipid amphotericin $\mathrm{B}$ and posaconazole is a grade CIII recommendation. Combination therapy with deferasirox is never recommended, grade $A 1 .^{20}$

Skiada et al. did not address recommendations for the diagnostic methods of mucormycosis infections. Mucormycosis is not detectable in the blood or CSF. There is not a blood antigen test or blood PCR detection that exists to detect mucormycosis, such as galactomannan and 1,3 beta D-glucan that identifies invasive aspergillosis. Biopsies with histopathological and culture evaluation are the best current method to detect mucormycosis infections. Molecular techniques and DNAPCR can also aid in the diagnosis from tissue samples. $^{20}$

In 2014, Cornely et al. and the European Society of Clinical Microbiology and Infectious Diseases, and the European Confederation of Medical Mycology strongly recommended direct microscopy, histopathology, cultures, and imaging to be used as diagnostic tools to detect mucormycosis and determine the depth of the infection. It is also strongly recommended that liposomal or lipid complex amphotericin B with a minimum dose of $5 \mathrm{mg} / \mathrm{kg} /$ day be initiated as first-line therapy in addition to surgical debridement and the treatment of any underlying disease processes. Posaconazole $200 \mathrm{mg} /$ day QID is strongly recommended as a treatment in refractory cases. All treatments should be continued until all predisposing factors or underlying comorbidities are well-controlled, in addition to resolution of the infection as seen with imaging. ${ }^{21}$

These recommendations do not specifically address invasive mucormycosis, mucormycosis of the GI tract, or mucormycosis in immunocompetent individuals, all which were reported in our case. There are many open studies and areas that have yet to be addressed in these papers that provide an avenue for future recommendations, guidelines, and specific protocols in these cases.

Currently, amphotericin B is the standard antifungal treatment used in mucormycosis and has shown to be effective in $100 \%$ of Rhizopus spp, Lichtheimia spp, and Rhizomucor spp. It has been found to be $94 \%$ effective against Mucor spp, with a MIC $<1$ $\mathrm{ug} / \mathrm{mL}$. Posaconazole has been found to have variable activity against of Rhizopus spp, Rhizomucor spp, and Mucor spp with $64-80 \%$ activity with a $0.5 \mathrm{ug} / \mathrm{mL}$. Other antifungals, such as fluconazole, voriconazole, itraconazole, echinocandins, flucytosine, terbinafine are not currently supported for use in the treatment of mucormycosis. ${ }^{20}$ Cresemba (isavuconazonium sulfate), ergosterol inhibitor, was recently approved for the treatment of invasive mucormycosis and aspergillosis that offers an extended spectrum antifungal activity with promising early study results. ${ }^{22.23}$ These findings provide an avenue for more research into GI mucormycosis in immunocompetent adults in the future, as well as for guidelines for adequate treatment.

This case has several interesting points. i) The patient was a relatively young immunocompetent woman with no previous medical history. ii) The mucor infection, which ultimately she succumbed too, developed at a distant site from the area affected at initial clinical presentation. iii) The source, time of exposure, and matter of transmission of the Mucor infection is not known. Inhalation, ingestion, and exposure to contaminated materials, both from outside or from within the hospital, are all possibilities. iv) Blood, sputum, nasal mucosa, and urine cultures were all negative. The diagnosis was made from a tissue sample. An abdominal tissue culture became positive for Rhizopus two weeks post-mortem.

Based on recognition of the above points, the following recommendations can be made.

In patients who have been treated with steroids, who have negative cultures, and who are not responding to treatment, clinicians should remain aware of the possibility of invasive fungal infections, including mucor, even in immunocompetent individuals.

Rapid diagnosis is essential to improve outcomes and chances for survival. Traditional tissue culture is problematic, primarily because of delays due to the time required for growth. Obtaining a tissue specimen endoscopically prior to the necessity for surgical intervention for evaluation by pathology is optimal. In order to further expedite diagnosis, obtaining a frozen section should be considered.

\section{References}

1. Spellberg B. Gastrointestinal mucormycosis: an evolving disease. Gastroenterol Hepatol (NY) 2012;8:140-2.

2. Hosseini M, Lee J. Gastrointestinal mucormycosis mimicking ischemic colitis in a patient with systemic lupus erythematosus. Am J Gastroenterol 1998;93:1360-2.

3. Petrikkos G, Skiada A, Lortholary 0, et al. Epidemiology and clinical manifestations of mucormycosis. Clin Infect Dis 2012;54:S23-34.

4. Lewis RE, Kontoyiannis K. Epidemiology and treatment of Mucormycosis. Future Microbiol 2013;8:1163-75.

5. Mooney JE, Wanger A. Mucormycosis of the gastrointestinal tract in children: report of a case and review of the literature. Pediatr Infect Dis J 1993;12:872-6.

6. Ibrahim AS, Spellberg B, Walsh TJ, Kontoyiannis DP. Pathogenesis of 
mucormycosis. Clin Infect Dis
2012;54:S16-22.

7. Rammaert B, Lanternier F, Zahar JR, et al. Healthcare-associated mucormycosis. Clin Infect Dis 2012;54:S44-54.

8. Mignogna MD, Fortuna G, Leuci S, et al. Mucormycosis in immunocompetent patients: a case-series of patients with maxillary sinus involvement and a critical review of the literature. Int $\mathrm{J}$ Infect Dis 2011;15:e533-40.

9. Sharma MC, Gill SS, Kashyap S, et al. Gastrointestinal mucormycosis--an uncommon isolated mucormycosis. Indian J Gastroenterol 1998;17:131-3.

10. Carr EJ, Scott P, Gradon JD. Fatal gastrointestinal mucormycosis that invaded the postoperative abdominal wall wound in an immunocompetent host. Clin Infect Dis 1999;29:956-7.

11. Sakorafas GH, Tsolakides G, Grigoriades $\mathrm{K}$, et al. Colonic mucormycosis: an exceptionally rare cause of massive lower gastrointestinal bleeding. Dig Liver Dis 2006;38:616-7.

12. Shiva prasad BN, Shenoy A, Nataraj KS. Primary gastrointestinal mucormycosis in an immunocompetent person. J Postgrad
Med 2008;54:211-3.

13. Van sickels N, Hoffman J, Stuke L, Kempe K. Survival of a patient with traumainduced mucormycosis using an aggressive surgical and medical approach. J Trauma 2011;70:507-9.

14. Anand J, Ghazala K, Chong VH. Massive lower gastrointestinal bleeding secondary to colonic mucormycosis. Med J Malaysia 2011;66:266-7.

15. Ryan 0, Fröhlich S, Crotty TB, Ryan D. Rhizopus microsporus infection in an immunocompetent host: a case of immunoparalysis?. Anaesth Intensive Care 2012;40:367-8.

16. Lalwani S, Govindasamy M, Gupta M, et al. Gastrointestinal mucormycosis--four cases with different risk factors, involving different anatomical sites. Indian $\mathbf{J}$ Gastroenterol 2012;31:139-43.

17. Choi HL, Shin YM, Lee KM, et al. Bowel infarction due to intestinal mucormycosis in an immunocompetent patient. J Korean Surg Soc 2012;83:325-9.

18. Bini R, Addeo A, Maganuco L, et al. The role of surgery in a case of diffuse mucormycosis with haematemesis and gastric necrosis. Ann R Coll Surg Engl 2014;96:e31-3.

19. Machicado JD, Younes M, Wolf DS. A rare cause of gastrointestinal bleeding in the intensive care unit. Healthcare-associated mucormycosis. Gastroenterology 2014;146: 1136-7.

20. Johnson CB, Ahmeti M, Tyroch AH, et al. Gastric mucormycosis as a cause of lifethreatening upper gastrointestinal bleeding in a trauma patient. Am Surg 2010;76:E76-7.

21. Skiada A, Lanternier F, Groll AH, et al. Diagnosis and treatment of mucormycosis in patients with hematological malignancies: guidelines from the 3rd European Conference on Infections in Leukemia (ECIL 3). Haematologica 2013;98:492-504.

22. Cornely OA, Arikan-akdagli S, Dannaoui E, et al. ESCMID and ECMM joint clinical guidelines for the diagnosis and management of mucormycosis 2013. Clin Microbiol Infect 2014;20:5-26.

23. Pettit NN, Carver PL. Isavuconazole: a new option for the management of invasive fungal infections. Ann Pharmacother 2015 [Epub ahead of print] 